\title{
Mobile Sink Approach to Minimize Congestion in Wireless Sensor Networks
}

\author{
B.Lalitha ${ }^{1}$, S.K.Gayaz ${ }^{2}$ \\ Associate Professor, Department of ECE, S V College of Engineering, Tirupati, A.P, India ${ }^{1,2}$
}

\begin{abstract}
The proposed paper addresses the setback of congestion in wireless sensor networks by introducing a mobile sink to reduce congestion. Moreover, in the considered method data has to voyage a restricted number of hops to arrive at the mobile sink which helps to improve the energy consumption of the sensor nodes. Parameters like packet delay, packet loss and throughput are measured for estimation. Since, congestion sternly affects the performance of wireless sensor networks, it has to be minimized in order to boost the life span of a sensor network. The mobile sink approach used in the planned scheme is very much effective in minimizing the congestion in the sensor network. In this model, the sink is not stationary, but it is mobile. With the use of simulation, the utility of the considered scheme in terms of congestion minimization in wireless sensor network is shown.
\end{abstract}

KEYWORDS: congestion minimization; wireless sensor networks; mobile sink.

\section{INTRODUCTION}

The occurrence of congestion can be observed in diverse types of wired and wireless networks although sturdy routing algorithms are present. Congestion in wireless sensor networks (WSN) primarily occurs because of two reasons -- when numerous nodes want to transmit data through the same channel at a time or when the routing node fails to advance the received data to the next routing nodes. Applications of WSNs need the sensor nodes to occasionally gather and route data towards a sink. It is also known that each sensor node can only be outfitted with a restricted quantity of storage, so if at any given routing node the data gathering rate dominates the data forwarding rate congestion tarts to assemble at this node. Such type of congestion and data loss occurs at the nodes located in the vicinity of static sink. Data failure at these nodes occurs as at any given position of time a sink can barely communicate restricted number of sensor nodes.[1][2][8][20]

To diminish the problems caused by static sink, new strategies are developed by making the productive use of the sink mobility to balance the trouble of congestion and the energy utilization among the sensors. That is, the mobile sink traverses the monitoring region and relax at several locations to collect sensed data. [1][2][3][7][8][20] The foremost part of this paper is that we have done the analysis on the effect of mobile sink in tumbling congestion by taking into consideration a range of parameters. The same parameters are taken into consideration for the sensor network with static sink. It has been discovered that, sensor network deploying a mobile sink has better performance than sensor network with a static sink to control congestion. The rest of the paper is structured as given below: Section 2 encapsulates related work, Section 3 describes the sink mobility model, simulation setup and investigation of results are specified in Section 4 and 5 respectively and section 6 concludes the paper.

\section{RELATED WORK}

In this section an abstract of presently existing congestion control techniques and their exclusion is discussed. It also extends the fresh work done on analyzing and finding the use of mobile sink in WSNs.

\section{Congestion Control Techniques}

In WSN, basically there are two main reasons that causes congestion- (i) the packet advent rate larger than the packet overhaul rate. This occurs at sensor nodes which are available close to sink as they cart extra upstream traffic. ii) Contention, interference and bit error rate on a link also causes congestion.[1][7][8][20] Congestion directly affects energy efficiency and may result buffer overflow, packet loss and can also corrupt link operation. Thus, congestion in WSN must be capably controlled. [1][20] Chen [13] classified the congestion control techniques in WSN into two groups: congestion avoidance and congestion control. The earlier part focus on strategies to avoid congestion from occurring and the second works on removing congestion when it has occurred. These techniques can be divided into below given three groups: data aggregation techniques, flow control techniques, and multi hop/path routing techniques.[1][13][20]

Data aggregation techniques: It focuses on using spatial or temporal correlation connecting sensed data to diminish the quantity of congestion.[1][19][20] 
Flow control techniques: It tries to control the magnitude of data that is flowing on the routing path to shun congestion using a variety of strategies[1][19][20]

Multi hop/path routing techniques: It utilizes the crowded employment of the sensor nodes to eliminate congestion from WSNs.[1][19][20] part from the above mentioned schemes Wang [14] projected a node precedence based congestion control method for wireless sensor networks. The method is based on the supposition that the nodes situated in a WSN have divergent bandwidth and wireless media control prerequisite for data transmission. Thus, a node priority index can be generated on the basis of packet inter arrival time and service time at each node. Using this index, sensor nodes with heavy data traffic can be assigned extra access to the transmission media than the nodes with fewer traffic. Nevertheless, extra overhead is implicated in this scheme for maintaining the priority index of the sensor nodes.[1][14][20] Investigation of the given schemes has shown that even though they diminish congestion, but they fall short to shun data and energy loss throughout this method. [2][4][7] To summarize, existing congestion control schemes escort data loss. In order to conquer this difficulty, a WSN with a mobile sink is planned in this paper. Now, various types of sink mobility models for data routing towards a mobile sink is discussed in the next section.

\section{DATA ROUTING TOWARDS A MOBILE SINK}

The use of a mobile sink has blown up in WSNs to get enhanced performance from the past few years for balanced employment of the sensor field energy. The sink in WSN can follow below given three basic types of mobility patterns:

A. Random mobility: Chatzigiannakis [16] used random sink mobility in their techniques. In case of random mobility, the sink follows a random path in the sensor field and implements a pull strategy in order to collect data from the sensor nodes. Alternatively, with random sink mobility it is impossible to guarantee data collection from all the sensor nodes situated in a WSN.[1][16][20]

B. Predictable mobility: Luo [17] have tried to discover a mobility approach for the sink that can lead to the most energy efficient employment of the sensor field. The best ever duration for the sensor network can only be achieved if the mobility route of the sink is along the outside edge i.e periphery of the sensor field. Enhanced data latency and packet loss are chief troubles that take place due to the sink mobility in wireless sensor networks. One major deficiency of this scheme is that every time the sink moves, routing paths need to be updated. [1][17][20]

C. Controlled mobility: Controlled sink mobility is analyzed by Jayaraman [18] in WSNs for escalating the lifetime.Schemes based on controlled sink mobility are a fine option if compressed data latency is obligatory, however they are less cost efficient than random/predictable mobility. [1][18] To sum up, if data latency is allowable, then the best routing policy that incurs least amount of data failure due to sink mobility and also provides highest lifetime of sensor network ith least cost is obtained if the sink follows a discrete mobility pattern along the periphery of the sensor field.[2][9]

\section{SIMULATION ENVIRONMENT}

In the planned model, 22 nodes are considered out of which 21 nodes are Wireless Sensor nodes and 1 node is a sink node. The wireless sensor nodes are equivalently but randomly deployed. Nodes are answerable for sensing and reporting their readings at continuous time to the sink. Sensor nodes are grouped into clusters and each cluster has a cluster head that is accountable for forwarding the expected data from adjacent nodes towards sink. The below given parameters are considered for wireless sensor network using a static sink in addition to wireless sensor networks using a mobile sink. The performance metrics like packet loss, packet delay as well as throughput have been chosen to evaluate the effect of mobile sink to lessen congestion in sensor network. The brief explanation and comparison is given in section 5. Further we show that, wireless sensor network using a mobile sink is proficient in minimizing the congestion and thus escalating the life span of the wireless sensor network.

Fig. 1 shows the simulation Environment which consists of 22 nodes out of which 21 nodes are WSN nodes and one node is a sink node. The same environment is considered for both static as well as mobile sink scenarios and the above performance metrics are considered for the comparison. Later we present the graphical analysis of both the scenarios. 


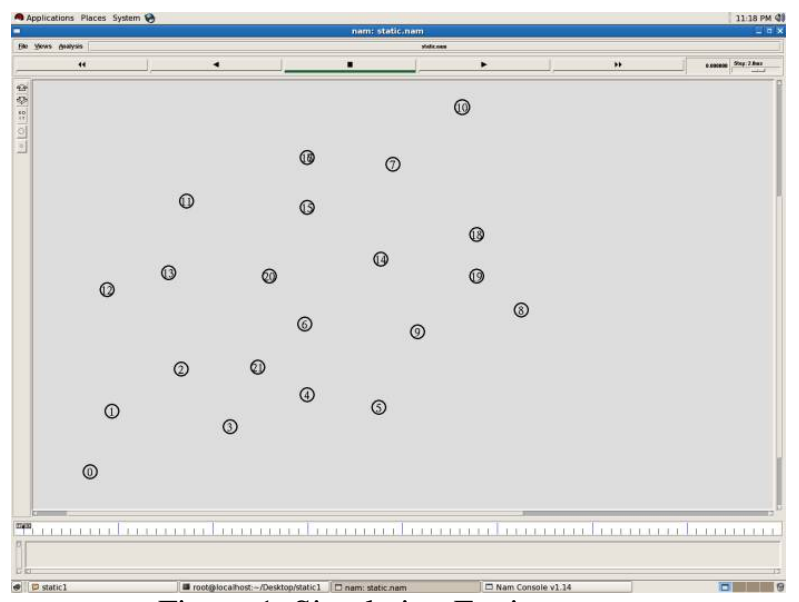

Figure 1. Simulation Environment

\section{RESULT ANALYSIS}

Besides running autonomously, both the simulations are proficient to obtain the graphs which depicts the packets which are lost in the network, packet delay and throughput. It can be observed from the simulation results that in a wireless sensor networks using a static sink, the nodes which are located in near to static sink, gets congested earlier as it is heavily loaded due to the fact that it is responsible for carrying the traffic of the neighboring nodes. Once congestion starts to upsurge in the network, the battery of the congested node drains out quickly, which ultimately results in node failure.

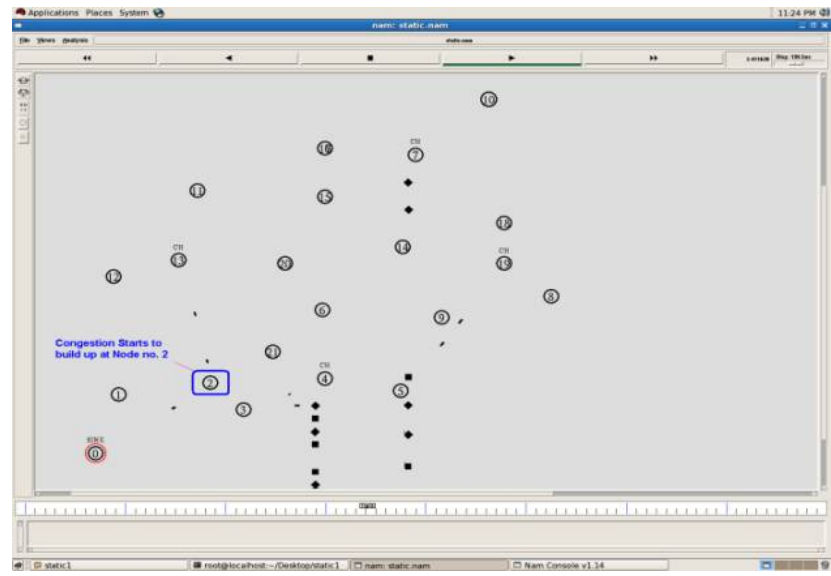

Figure 2. Congestion starts to upsurge at node 2 which is located near to Static Sink.

\section{CONCLUSION}

The Simulation results obtained from the proposed model, we conclude that, congestion, which is a foremost factor disturbing the performance of a Wireless Sensor Network, has condensed significantly by using a Mobile sink. Also, congestion has a direct impact on the life span of the sensor network. By scheming congestion, we are able to boost the life span of the sensor network.

\section{REFERENCES}

[1] Sagar Motdhare, C. G. Dethe, "Congestion Avoidance and Lifetime Maximization in Wireless Sensor Networks using a Mobile Sink ", In the proceedings of ninth international conference on Wireless Communications and Sensor Networks, Vol 299 of Lecture notes in Electrical Engineering, pp 37-49, April 2014

[2] Majid I Khan, Wilfried N Gansterer, Gunter Haringm, "Congestion avoidance and energy efficient routing protocol for WSN with mobile sink."In the journal of Networks, Vol 2, no. 6, December 2007.

[3] Majid I. Khan, Wilfried N. Gansterer, Guenter Haring, "Innetwork storage model for data persistence under congestion in wireless sensor network". In Proceedings of the First International Conference on Complex, Intelligentand Software Intensive Systems (CISIS'07), 2007, pp. 221228.

[4] Wan, C., Eisenman, S. B., and Campbell, A. T. "CODA: congestion detection and avoidance in sensor networks". In Proceedings of the $1^{\text {st }}$ international Conference on Embedded Networked Sensor Systems (Los Angeles, California, USA, November 05 - 07, 2003). SenSys '03. ACM Press, New York, NY, pp. 266-279.

[5] Siddharth Ramesh, "A Protocol Architecture for WSN", School of Computing, university of Utah, Salt Lake City, UT 84112. 